Ann. Biol. anim. Bioch. Biophys., 1976, 16 (6), 795-805.

\title{
RELATION ENTRE LES PROTÉINES DE LIAISON DE LA PROGESTÉRONE ET LA CONCENTRATION PLASMATIQUE PÉRIPHÉRIQUE DE PROGESTÉRONE CHEZ GALLUS DOMESTICUS
}

\author{
Catherine MONET (1), B. MARTIN et M. THIBIER* \\ Laboratoire de Physiologie de la Reproduction, \\ Université $P$. et $M$. Curie, \\ 12 , rue Cuvier, \\ 75005 Paris \\ * Institut national agronomique, Paris-Grignon, \\ 78850 Thiverval-Grignon
}

\section{RÉSUMÉ}

La liaison des stéroïdes $\mathrm{C}_{21}$ aux protéines plasmatiques a été étudiée chez Gallus domesticus. La progestérone et la corticostérone se lient avec une grande affinité $\left(K_{A} \simeq 5\right.$. Io $\left.{ }^{8} \mathbf{M}^{-1}\right)$ et une capacité de liaison limitée $\left(N \simeq 2 \cdot I^{-7} \mathrm{M}\right.$ ) aux mêmes systèmes protéiques. Les constantes de liaison ne sont pas significativement différentes entre les poules " standard " ou naine et le coq. Les taux plasmatiques de progestérone de la poule standard et de la poule naine varient entre 0,3 et $\mathrm{I}, 2 \mathrm{ng} / \mathrm{ml}$, quelques heures après la ponte. Chez le coq, ils sont inférieurs à la limite de détection. Aucune corrélation entre le taux plasmatique de progestérone et la capacité de liaison de la progestérone aux protéines n'a été observée chez la Poule.

\section{INTRODUCTION}

Divers auteurs (FuRR et al., I973; LAGUË et al., I975; Shahabi et al., I975; SHoDono et al., I975) ont mis en évidence des variations de la concentration plasmatique des stéroìdes chez Gallus domesticus. Pour tenter de préciser l'importance physiologique de ces variations, il est nécessaire de connaître les éventuels systèmes de liaison de ces stéroïdes aux protéines du plasma. Il semble en effet, à la lumière de diverses observations faites chez plusieurs Mammifères que les stéroïdes complexés aux protéines ne sont pas biologiquement actifs (WESTPHAL, I97I).

La présence d'un système protéique plasmatique capable de lier spécifiquement

( ${ }^{2}$ Adresse actuelle : Station de Recherches sur la Physiologie de la Reproduction, Centre de Recherches de Tours, I. N. R. A., Nouzilly, 37380 Monnaie, B.P. I. 
la corticostérone et la progestérone a été clairement démontrée chez plusieurs vertébrés. Dans les différentes espèces d'Oiseaux étudiées, la corticostérone, qui est le principal corticostéroïde sécrété par les surrénales (SANDOR, 1972) se fixe aux protéines plasmatiques (Steeno et De Moor, I966; Gould et SiEgel, I974; SEAL et Doe, I965; Corvol, et BARdin, I973; DANIEL et ASSENMAChER, I974; MARTIN, I974). Sherman et al. (I970) et MARTin (I974) ont montré également que la progestérone pouvait se fixer spécifiquement à ces protéines. Les caractéristiques de liaison de la progestérone et de la corticostérone aux systèmes protéiques plasmatiques ont été établies dans l'espèce humaine (transcortine ou CBG), et pour certains Mammifères (" transcortin type ") et Amphibiens ("Corticosteroïd Binding Protein " ou CBP) (DAUGHaday, I956, I958 ; SEAL, et DoE, I965; MARTin et OzON, I975).

Cette étude réalisée chez Gallus domesticus a pour but : I) de caractériser les systèmes protéiques plasmatiques de liaison de la corticostérone et de la progestérone ; 2) de déterminer la concentration de progestérone dans le plasma de poules "standard" de poules naines et de coqs ; 3) d'analyser les variations quantitatives des protéines de liaison au cours de différents états physiologiques (sexe et taille). Nous nous proposons en outre de relier le taux plasmatique de ces protéines liant la progestérone à la concentration plasmatique périphérique de ce stéroïde.

\section{MATÉRIEL E'T MÉTHODES}

\section{Animaux}

Cette étude a été réalisée à partir de prélèvements sanguins de ro coqs adultes, de 8 poules. pondeuses de taille normale "standard " et de 12 poules naines pondeuses provenant de la Station de Génétique Factorielle de l'I.N.R.A. (Jouy en Josas), les poules ayant pondu quelques heures avant le prélèvement. Le sang prélevé dans la veine alaire a été immédiatement centrifugé, le plasma décanté et congelé a $-20^{\circ} \mathrm{C}$. Dans certains cas, du sérum a été obtenu ; la centrifugation a été effectuée I 2 heures environ après le prélèvement. Certaines déterminations ont été réalisées après adsorption des stéroïdes endogènes par le charbon Norit A (HeYNes et al., I967). La concentration protéique a été déterminée à partir de la formule de KaLckAR (I947).

\section{Stéroides}

Les stéroïdes utilisés étaient chromatographiquement purs et provenaient du commerce (NEN, Amersham, Roussel-Uclaf) : $1,2{ }^{3} \mathrm{H}$-corticostérone, AS : $50 \mathrm{Ci} / \mathrm{mmole} ; \mathrm{I}, 2,6,7^{-3} \mathrm{H}$-progestérone, AS $81 \mathrm{Ci} / \mathrm{mmole} ; 1,2^{-3} \mathrm{H}$-progestérone, AS : $53 \mathrm{Ci} / \mathrm{mmole}$ ).

La détermination quantitative de la radioactivité a été réalisée sur spectrophotomètre à scintillation liquide Packard "Tricarb ", modèle 3320, avec standard externe. Les mesures sont effectuées à partir d'un mélange formé par $0,5 \mathrm{ml}$ de l'échantillon étudié et Io $\mathrm{ml}$ du mélange $P P O$ $(5,5 \mathrm{~g})$, POPOP $(0, \mathrm{I} \mathrm{g})$, Triton $\times$-IOO $(333 \mathrm{ml})$, toluène $(667 \mathrm{ml})$ (PatTerson et Green, I965).

\section{Incubations}

L'extrait à étudier a été incubé à $4^{\circ} \mathrm{C}$ pendant 20 heures avec de la progestérone dont la concentration variait de $\mathrm{IO}^{-10} \mathrm{M}$ à $\mathrm{ro}^{-7} \mathrm{M}$, suivant les types d'expériences.

\section{Détermination de la liaison stéroïde-protéine}

1. Filtration sur gel de Séphadex G-25.

Les colonnes $(h=9 \mathrm{~cm} ; \varnothing=\mathrm{I} \mathrm{cm})$ sont équilibrées à $4^{\circ} \mathrm{C}$ avec un tampon $T r i s-\mathrm{HCl}(\mathrm{o}, \mathrm{OI} \mathrm{M})$ $\mathrm{NaCl}\{0,09 \mathrm{M}), \mathrm{pH} 8$. L'extrait $(0,2 \mathrm{ml}$; I $\mathrm{mg}$ de protéines par $\mathrm{ml})$ est passé sur chaque colonne 
(débit $20 \mathrm{ml} / \mathrm{h}$ ) avec un mélange de bleu Dextran (Pharmacia) et de rouge de chlorophénol. La fraction liée est éluée avec le bleu Dextran et la fraction libre avec le rouge de chlorophénol. La fraction bleue est recueillie et la radioactivité mesurée.

\section{Dialyse à l'équilibre.}

Les sacs de dialyse (Arthur $\mathrm{H}$. Thomas Company) sont préalablement lavés avec le tampon de dialyse (Tris-HCl o,or M, pH 7,4) pendant 24 heures à $4^{\circ} \mathrm{C}$. Pour toutes les dialyses réalisées; I $\mathrm{ml}$ de plasma dilué (au $\mathrm{I} / 20, \mathrm{I} / 40$ et $\mathrm{I} / 80$ ) avec le tampon de dialyse est placé à l'intérieur du sac, le stéroïde radioactif étant à l'extérieur dans $4 \mathrm{ml}$ de tampon. Pour les expériences de compétition, le stéroïde non radioactif a été mis avec le stéroïde radioactif à l'extérieur du sac. A chaque dialyse ont été ajoutées des quantités variables de stéroïde non radioactif ( $\mathrm{ro}^{-10} \mathrm{M}$ à $\mathbf{r o}^{-7} \mathrm{M}$ ) et une quantité fixe de stéroïde radioactif $\left(\mathrm{IO}^{-13} \mathrm{M}\right)$. L'équilibre de la dialyse est atteint au bout de $4^{8}$ heures à $4^{\circ} \mathrm{C}$ sous agitation constante. Le pourcentage de fixation du stéroïde lié aux protéines est calculé à l'équilibre de dialyse : pourcentage de liaison $=\mathrm{x} 00[\mathrm{I}-(\mathrm{D}$. Vr $) /(\mathrm{R}$. Vd) $]$, où $\mathrm{R}$ et $\mathrm{D}$ sont les quantités totales de radioactivité à l'intérieur et à l'extérieur du sac de dialyse, et $\mathrm{Vr}$ et Vd sont les volumes correspondants (SANDBERG et al., I966).

\section{Electrophorèse sur gel de polyacrylamide.}

L'analyse électrophorétique de plasma de poule a été réalisée sur gel de polyacrylamide 7.5 p. Ioo dans un tampon Tris-glycine $\mathrm{pH} \mathrm{8,3}$ (DAvis, I962). La ${ }^{3} \mathrm{H}$-progestérone (Io ${ }^{-8} \mathrm{M}$ ) a été ajoutée au gel avant polymérisation. L’extrait (90o $\mu \mathrm{g}$ de protéines) préalablement incubé avec ${ }^{3} \mathrm{H}$-progestérone $\left(5 \times \mathrm{IO}^{-8} \mathrm{M}\right.$ ) est déposé sur le gel et soumis à l'électrophorèse ( $120 \mathrm{mn}$ à $0^{\circ} \mathrm{C}$; I,5 $\mathrm{mA}$ par tube). Après électrophorèse, le gel est refroidi à $-25^{\circ} \mathrm{C}$, coupé en tranches de $\mathrm{I} \mathrm{mm}$ et la radioactivité comptée.

\section{Paramètres de liaison}

Les constantes de liaison - capacité de liaison $(\mathrm{N})$ et constante de dissociation apparente $\left(K_{D}\right)$ - ont été calculées par une méthode itérative de minimalisation au sens des moindres carrés (RAYNAUD, I973) et par une méthode graphique de type ScatchaRd (I949) modifiée par RosenTHAL (1967). Des résultats identiques ont été obtenus par ces deux méthodes. Les valeurs calculées tiennent compte de la présence de stéroüdes endogènes dans les différents échantillons. La fixation non saturable est quantifiée par la valeur $\mathrm{K}_{\mathrm{N}} \mathrm{N}_{\mathrm{N}} \mathrm{s}$ qui correspond au produit de la constante d'association de la liaison par la concentration en sites de liaison non saturables dans nos conditions expérimentales.

\section{Dosage de la progestérone plasmatique}

Les dosages ont été effectués selon la méthode décrite par Thisier et al. (1973). Elle comprend une purification partielle par chromatographie sur microcolonne de Séphadex LH 20 et une séparation des stéroïdes libres et liés à l'anticorps par le toluène. L'antisérum a été préparé sur lapin par injection de progestérone-I I $\alpha$-hémisuccinate-Bovine Serum Albumin.

\section{RÉSULTATS}

\section{A. - Identification des systèmes plasmatiques de liaison de la progestérone et de la corticostérone}

\section{Spécificité de liaison de la progestérone et de la corticostérone aux protéines plasma- tiques de coq.}

La spécificité de la liaison stéroïdes $\mathrm{C}_{21}$-protéines plasmatiques a été étudiée à partir de plasma de coq (dilué 20 ou 40 fois) par équilibre de dialyse en utilisant la méthode des compétitions. 
Des expériences de compétition ont été réalisées avec ${ }^{3} \mathrm{H}$-progestérone et ${ }^{3} \mathrm{H}$ corticostérone $\left(\mathrm{IO}^{-10} \mathrm{M}\right)$, en augmentant progressivement les concentrations de ces mêmes stéroïdes (non radioactifs, $\mathbf{I O}^{-10} \mathrm{M}$ à $\mathrm{IO}^{-7} \mathrm{M}$ ). Les degrés de compétition de la progestérone $v s{ }^{3} \mathrm{H}$-progestérone et de la corticostérone $v s{ }^{3} \mathrm{H}$-corticostérone ont été utilisés comme référence. Il apparaît (fig. I) que le complexe ${ }^{3} \mathrm{H}$-corticostérone est aussi fortement déplacé par la corticostérone que par la progestérone, et que le complexe ${ }^{3} \mathrm{H}$-progestérone est aussi fortement déplacé par la progestérone que par la corticostérone. Il est important de noter en outre que la fixation non saturable de ${ }^{8} \mathrm{H}$-progestérone est plus élevée que celle obtenue pour ${ }^{3} \mathrm{H}$-corticostérone (fig. I).
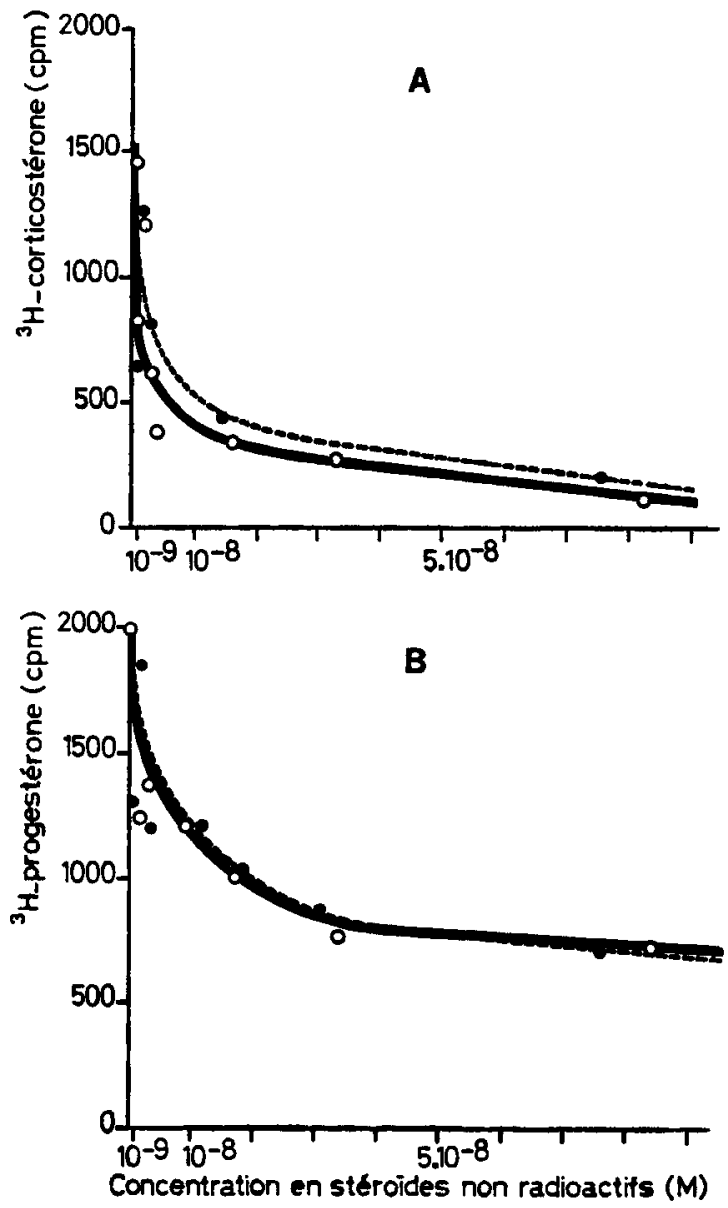

FIG. I. - Etude de la fixation de ${ }^{3} \mathrm{H}$-corticostérone (A) et ${ }^{3} \mathrm{H}$-progestérone (B) aux protéines plasmatiques de coq en fonction des concentrations en progestérone $(\mathrm{O}-\mathrm{O})$ et corticostérone (---O) non radioactives. Dialyses à l'équilibre avec du plasma dilué 20 fois.

Le tableau I montre que les stéroïdes étudiés peuvent être classés en deux groupes suivant leur modalité de fixation : I) ceux entrant en compétition avec la progestérone dès la plus faible concentration utilisée $\left(\mathrm{I}^{-9} \mathrm{M}\right)$, et dont le degré de compétition 
est proportionnel à leur concentration (groupe I), et 2) ceux qui n'entrent en compétition avec la progestérone qu'à de fortes concentrations ( Io $^{-6} \mathrm{M}$ ) (groupe 2). Les faibles déplacements de la liaison ${ }^{3} \mathrm{H}$-progestérone-protéines observés en présence de progestérone froide sont dus à la iorte fixation de l'hormone aux protéines plasmatiques non saturables.

TABLEAU I

Effets compétitifs de différents stéroides

sur le complexe ${ }^{3} \mathrm{H}$-progestérone-protéines plasmatiques de coq

\begin{tabular}{|c|c|c|c|}
\hline & $10^{-9} \mathrm{M}$ & $10^{-8} \mathrm{M}$ & $10^{-6} \mathrm{M}$ \\
\hline \multicolumn{4}{|l|}{ Groupe 1} \\
\hline Progestérone . . . . . . . . . . . & - & 86 & 66 \\
\hline $17 \alpha$-hydroxyprogestérone $\ldots \ldots \ldots$ & 93 & 83 & 68 \\
\hline $16 \alpha$-hydroxyprogestérone $\ldots \ldots \ldots$ & 86 & 85 & 75 \\
\hline $5 \beta$-pregn-3,20-dione . . . . . . . . . & 96 & 89 & 68 \\
\hline $5 \alpha$-pregn-3,20-dione $\ldots \ldots \ldots \ldots \ldots$ & 97 & 86 & 70 \\
\hline Désoxycortisostérone $\ldots \ldots \ldots \ldots$ & 92 & 90 & 67 \\
\hline Corticostérone $\ldots \ldots \ldots \ldots \ldots \ldots$ & 92 & 86 & 77 \\
\hline Cortisol $\ldots \ldots \ldots \ldots \ldots \ldots$ & 94 & 82 & 69 \\
\hline Cortisone $\ldots \ldots \ldots \ldots \ldots \ldots \ldots$ & 94 & 73 & 68 \\
\hline \multicolumn{4}{|l|}{ Groupe 2} \\
\hline $20 \alpha$-hydroxyprogestérone $\ldots \ldots \ldots$ & 100 & 99 & 71 \\
\hline 20ß-hydroxyprogestérone ........ & 98 & 98 & 70 \\
\hline $5 \alpha$-pregn- $3 \beta, 20 \beta$-diol $\ldots \ldots \ldots \ldots$ & 100 & 100 & 83 \\
\hline Pregnénolone $\ldots \ldots \ldots \ldots \ldots \ldots$ & 100 & 99 & 78 \\
\hline $3 \beta$-hydroxy-5 $\alpha$-pregn-20-one $\ldots \ldots$. & 100 & 95 & 67 \\
\hline $3 \alpha$-hydroxy-5 $\beta$-pregn-20-one $\ldots \ldots$ & 100) & 100 & 83 \\
\hline Dexaméthasone $\ldots \ldots \ldots \ldots \ldots$ & 95 & 96 & 65 \\
\hline Androsténedione $\ldots \ldots \ldots \ldots \ldots$ & 97 & 94 & 78 \\
\hline Testostérone ............ & 100 & 100 & 70 \\
\hline Gstradiol-17 $\alpha \ldots \ldots \ldots \ldots \ldots$ & 99 & 97 & 84 \\
\hline Estradiol-17 $\beta \ldots \ldots \ldots \ldots \ldots \ldots$ & 95 & 92 & 86 \\
\hline
\end{tabular}

Les expériences de compétition sont réalisées par équilibre de dialyse en présence de $6 \cdot 10^{-9} \mathrm{M}$ de ${ }^{3} \mathrm{H}$-progestérone et de stéroïdes non radioactifs à différentes concentrations $\left(10^{-9} \mathrm{M}, 10^{-8} \mathrm{M}, 10^{-6} \mathrm{M}\right)$. Le plasma est dilué au $40^{\mathrm{e}}$. Les résultats sont exprimés sous forme de liaison relative de la progestérone tritiée aux protéines. La valeur arbitraire 100 correspond à la fixation de ${ }^{3} \mathrm{H}$-progestérone aux protéines plasmatiques lorsque le plasma est mis uniquement en présence de $6 \cdot 10^{-9} \mathrm{M}$ de ${ }^{3} \mathrm{H}$-progestérone.

2. Constantes de liaison apparentes de la progestérone et de la corticostérone aux protéines plasmatiques.

La détermination des constantes de liaison par équilibre de dialyse montre que la corticostérone et la progestérone se fixent avec une grande affinité $\left(\mathrm{K}_{\mathrm{A}} 4^{\circ} \mathrm{C} \simeq 5 \cdot \mathrm{ro}^{8}\right.$ $\left.\mathrm{M}^{-1}\right)$ et une capacité de liaison limitée $\left(\mathrm{N} \simeq 2 \cdot \mathrm{IO}^{-7} \mathrm{M}\right)$ aux protéines plasmatiques de poule et de coq. Les constantes de liaison apparentes de la corticostérone et de la 
progestérone aux protéines du plasma de poule sont rapportées dans le tableau 2. La corticostérone et la progestérone se fixent avec le même $K_{D}$ aux protéines plasmatiques $(p>0,05)$. Les capacités de liaison $(\mathrm{N})$ de ces deux stéroïdes aux protéines ne sont pas significativement différentes $(p>0,05)$. En revanche, si 1'on quantifie la part de liaison non spécifique par le $\mathrm{K}_{\mathrm{N}} \mathrm{N}_{\mathrm{NS}}$, il apparaît que la fixation de la progestérone aux protéines non spécifiques est plus grande que celle observée pour la corticostérone.

\section{TABLEAU 2}

Consiantes de dissociation $\left(\mathrm{K}_{\mathrm{D}}\right)$, capacités de liaison $(\mathrm{N})$ et constantes de liaison non spécifique $\left(\mathrm{K}_{\mathrm{N}} \mathrm{N}_{\mathrm{NS}}\right)$ de la progestérone et de la corticostérone aux protśines plasmatiques de la Poule fondeuse

\begin{tabular}{|c|c|c|c|c|}
\hline \multirow{2}{*}{$\begin{array}{l}\text { Nombre } \\
\text { d'ani- } \\
\text { maux }\end{array}$} & \multirow{2}{*}{ Stéroïde } & \multicolumn{3}{|c|}{ Moyenne \pm écart-type } \\
\hline & & $K_{D}\left(10^{-9} M\right)$ & $\mathrm{N}\left(10^{-7} \mathrm{M}\right)$ & $\mathrm{K}_{\mathrm{NS}} \mathrm{N}_{\mathrm{NS}}$ \\
\hline 4 & Corticostérone & $2,64 \pm 1,60$ & $2,01 \pm 1,32$ & (a) $0,09 \pm 0,10$ \\
\hline 7 & Progestérone & $2,36 \pm 1,22$ & $2,07 \pm 0,62$ & (b) $0,63 \pm 0,12$ \\
\hline
\end{tabular}

(a) et $(b)$ : Valeurs significativement différentes $(p<0,01)$.

La séparation sur colonne de Séphadex G-25 des stéroïdes liés et des stéroïdes libres donne une valeur du $N$ légèrement inférieure (pour la progestérone, $N=I, 44 \times$ $\left.\mathrm{IO}^{-7} \mathrm{M}\right)$.

Lorsque les plasmas de poule ou de coq sont saturés en corticostérone ( $\left.1 \mathrm{r}^{-4} \mathrm{M}\right)$ pendant 20 heures, la progestérone ne se fixe plus spécifiquement aux protéines plasmatiques; la faible fixation de la progestérone correspond à une fixation non spécifique.

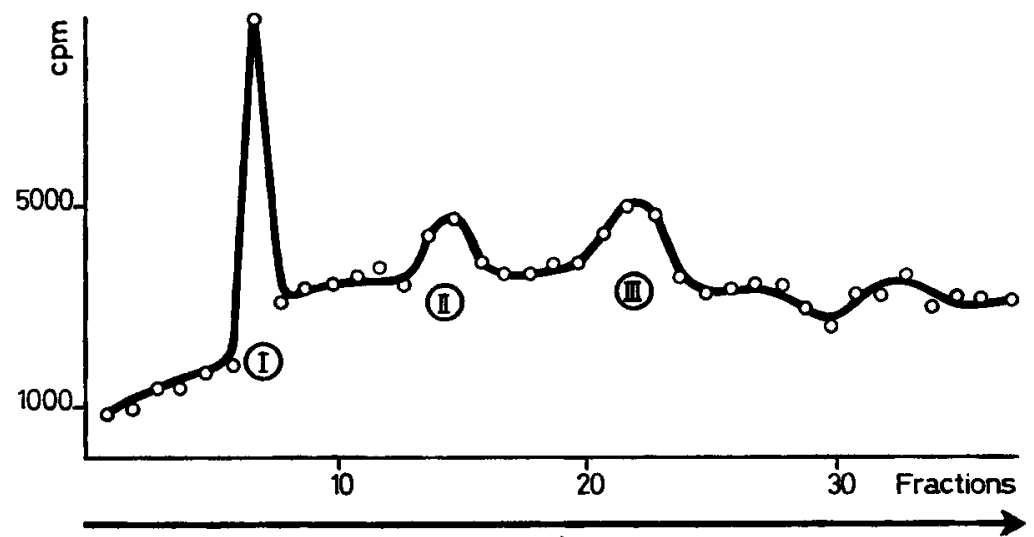

FIG. 2. - Electrophorèse sur gel de polyacrylamide de plasma de poule incubé avec ${ }^{3} H$-progestêrone. La flèche indique le sens de la migration. ${ }^{3} \mathrm{H}$-progestérone a été ajoutée au gel avant polymérisation. L'extrait a été préalablement incubé avec ${ }^{3} \mathrm{H}$-progestérone $\left(5 \times 10^{-8} \mathrm{M}\right)$ puis déposé sur le gel de polyacrylamide (7,5 p. 100). 
Les valeurs des constantes de liaison de la progestérone aux protéines obtenues à partir de sérum ne diffèrent pas significativement de celles obtenues à partir de plasma $(p>0,05)$.

\section{Analyse électrophorétique des protéines de liaison de la progestérone.}

Le comportement électrophorétique sur gel de polyacrylamide du plasma de poule marqué avec ${ }^{3} \mathrm{H}$-progestérone, confirme l'existence d'une fixation de la progestérone aux protéines. Les complexes ${ }^{3} \mathrm{H}$-progestérone-protéines (fig. 2) se répartissent dans 3 fractions protéiques principales (I, II et III) que nous n'avons pas cherchées à identifier.

\section{B. - Étude des variations quantitatives des protéines de liaison de la progestérone en fonction de différents états physiologiques}

D'après l'examen du tableau 3 il semble que les valeurs des constantes de liaison sont plus élevées pour la poule " standard " que pour la poule naine et le coq. Cependant les estimations moyennes de la capacité de liaison ou de la constante de dissociation ne sont pas significativement différentes entre les 3 groupes $(p>0,05)$, en raison d'une grande variabilité entre individus de chacun des groupes (coefficients de variation compris entre 40 et $95 \mathrm{p}$. Ioo).

TABLEAU 3

Constantes de liaison de la progestérone aux protéines plasmatiques de poule "standard", de poule naine et de coq

\begin{tabular}{c|c|c}
\hline \multicolumn{1}{c|}{ Animaux } & $\mathrm{N}\left(10^{-7} \mathrm{M}\right)$ & $\mathrm{K}_{\mathrm{D}}\left(10^{-\theta} \mathrm{M}\right)$ \\
\hline Poules a standard " (8) & $2,07 \pm 0,81$ & $2,58 \pm 1,37$ \\
\hline Poules naines (10) & $1,48 \pm 0,86$ & $2,26 \pm 2,12$ \\
\hline Coqs (7) & $1,31 \pm 0,67$ & $1,59 \pm 0,86$ \\
\hline
\end{tabular}

Moyenne \pm écart-type ; ( ) nombre d'animaux.

\section{C. - Concentrations plasmatiques de la progestérone}

La concentration plasmatique (moyenne et écart-type) est respectivement égale, chez les poules "standard " et naines, à $0,69 \pm 0,38$ et $0,79 \pm 0,21$ ng/ml (non significativement différentes; $p>0,05)$. Chez le coq, cette concentration est égale ou inférieure à la limite de détection $(0,05 \mathrm{ng} / \mathrm{ml}$. Elle est donc très notablement moindre que celle observée chez la Poule. 


\section{D. - Relation entre la concentration de progestérone plasmatique et la concentration des protéines liant la progestérone}

Le coefficient de corrélation entre le taux de progestérone et la capacité de liaison a été calculé pour l'ensemble des poules " standard" et naines. Il est non significatif $(p>0,05 ; n=9)$.

La capacité de liaison apparaît en outre, selon le sexe, comme une variable indépendante de la concentration de progestérone. En effet, bien que chez le mâle, la concentration en progestérone soit nettement inférieure à celle de la femelle, 1a concentration en sites de liaison n'est pas significativement différente de celle observée chez la Poule.

\section{DISCUSSION}

Les expériences de compétition ainsi que la détermination des constantes de liaison montrent que la progestérone et la corticostérone se lient avec une grande affinité $\left(\mathrm{K}_{\mathrm{A}} 4^{\circ} \mathrm{C}=5 \times 10^{8} \mathrm{M}^{-1}\right)$ et une capacité de liaison limitée $\left(\mathrm{N}=2 \times 10^{-7} \mathrm{M}\right)$ aux mêmes systèmes protéiques. La capacité de liaison de la progestérone aux protéines de poule déterminée par équilibre de dialyse $(6,3 \mu \mathrm{g} /$ Ioo $\mathrm{ml}$ de plasma) est supérieure à celle trouvée par filtration sur gel de Séphadex $(4,5 \mu \mathrm{g} /$ Ioo ml). Cette dernière valeur se rapproche de celles obtenues par SEAL, et DOE (I965) et GouLD et SIEGEL (I974) en utilisant la même technique (I,5-6 $\mu \mathrm{g} / \mathrm{IO0} \mathrm{ml}$ ). Les plus faibles valeurs des capacités de liaison obtenues après filtration sur gel, seraient dues à une grande dissociation des complexes stéroïdes $\mathrm{C}_{21}$-protéines au cours du fractionnement chromatographique.

Les expériences de compétition montrent que les stéroïdes se complexant aux protéines de Gallus domesticus avec une grande affinité, ont en commun une structure moléculaire de type pregnane $\left(C_{21}\right.$, avec $\Delta_{4}$ ou $5 \alpha$ ou $\left.5 \beta\right)$ et possèdent deux groupements cétoniques en positions 3 et 20 . L'existence d'un groupement hydroxyl en position $2 \mathrm{I}$ (désoxycorticostérone) ou en $\mathrm{I} 7 \alpha$ ( $\mathrm{I} 7 \alpha$-hydroxyprogestérone) ou en $\mathrm{I} 6 \alpha$ (I6 $\alpha$-hydroxyprogestérone) ou en II et 2I (corticostérone) ou en II, 2I et I7 $\alpha$ (cortisol) ne modifie pas la liaison. Il en est de même pour les composés $5 \alpha$ et $5 \beta$ réduits de la progestérone ( $5 \alpha$ et $5 \beta$-pregn-3, 20-dione). Par contre l'existence d'un groupement hydroxyl en position 20 (20 $\alpha$-hydroxyprogestérone ; $20 \beta$-hydroxyprogestérone) ou en position 3 ( $3 \alpha-\mathrm{OH}-5 \beta$-pregn-2o-one; $3 \beta-\mathrm{OH}-5 \alpha$-pregn-2o-one) ne permet pas cette liaison de haute affinité. Par ailleurs il est intéressant de noter que l'affinité de liaison de la testostérone et de l'œstradiol-I $7 \beta$ aux protéines de poule est du même ordre que celle observée avec la CBG humaine (DOE et SEAL, I963).

La présence de trois principales fractions protéiques liant la progestérone observées après électrophorèse sur gel de polyacrylamide de plasma de poule est à mettre en relation avec les résultats de GouLd et SrEGEL (I974). Ces derniers auteurs montrent en effet, par chromatographie sur échangeurs d'ions, au moins trois systèmes de liaison des stéroïdes $\mathrm{C}_{21}$ dans le plasma de Gallus domesticus. La caractérisation de ces protéines permettrait la mise en évidence éventuelle d'une protéine analogue à la CBG humaine. 
La concentration en protéines de liaison n'apparaît pas plus élevée dans un sexe que dans un autre. Le nombre relativement réduit d'animaux par groupes (I8 femelles et 7 mâles) associé à la variance importante de cette constante peut expliquer notre impossibilité à mettre en évidence une différence significative entre ces groupes d'animaux. STEENo et de Moor (I966) ne signalent pas non plus de différence dans les concentrations en protéines de liaison des corticostéroïdes suivant le sexe, chez le Canard (domestique ou de barbarie) et le Paon. SEAL et DOE (I965) ainsi que GouLD et SIEGEL, (I974) ont noté une plus grande capacité de liaison chez la Poule que chez le Coq. Cependant ces premiers auteurs n'ont effectué leur étude que sur 3 animaux de chaque sexe. En ce qui concerne les résultats des seconds auteurs, malgré la concentration en sites légèrement plus faible chez le coq, aucun test statistique ne confirme une différence éventuellement significative entre les 2 sexes. DANIEL et ASSENMACHER (I974) indiquent une concentration en protéines de liaison des stéroïdes $\mathrm{C}_{21}$ plus faible chez la Cane que chez le Canard (de Pékin).

Les taux plasmatiques de progestérone de la poule oscillent entre 0,3 et $\mathrm{I}, 2 \mathrm{ng} / \mathrm{ml}$. Ces valeurs ont été obtenues à partir de prélèvements sanguins réalisés quelques heures après la ponte du matin. Connaissant le cycle ovulatoire de la poule (in LoRENZ, I969), nous pouvons dire que les prélèvements ont été effectués quelques heures après une ovulation, donc à un moment où le taux de progestérone est à son niveau de base. Nos valeurs, obtenues tant chez la Poule " standard " que chez la Poule naine, sont en accord avec les valeurs des taux de base obtenues par FuRR et al. (I973), par Shahabi et al. (1975) et par Shodono et al. (1975) chez des poules Leghorn.

Les protéines plasmatiques peuvent lier une quantité de progestérone $(63 \mathrm{ng}$ de progestérone par $\mathrm{ml}$ de plasma) qui est roo fois supérieure environ à la quantité de progestérone plasmatique observée dans cette étude, et correspondant à un niveau basal, et Io fois supérieure à la quantité maximale de progestérone présente dans le plasma 4 à 6 heures avant l'ovulation $(\simeq 6 \mathrm{ng} / \mathrm{ml}$ ) (SHodono et al., I975). En tenant compte de la concentration plasmatique en corticostérone $(\simeq$ ro $\mathrm{ng} / \mathrm{ml}$ d'après GOULD et SIEGEL, I974), la concentration en protéines de liaison est encore largement suffisante pour lier spécifiquement et avec une haute affinité la progestérone et la corticostérone.

On n'observe chez la femelle aucune relation entre les taux de progestérone et de protéines de liaison plasmatiques. Les variations de concentration de progestérone sont ici relativement faibles car elles reflètent seulement les variations du niveau basal. Il est donc peu surprenant que la variation entre individus aduites du taux de protéines de liaison ne lui soit pas significativement corrélée.

Dans le plasma du jeune coq, FURR (I973) signale une concentration en progestérone de 1'ordre de $0,44 \mathrm{ng} / \mathrm{ml}$. Ce niveau est bien supérieur à celui de nos observations faites sur le coq adulte. Fin effet, nous n'avons relevé chez aucun coq une concentration supérieure à notre limite de détection estimée à $0,05 \mathrm{ng} / \mathrm{ml}$. Cependant les protéines plasmatiques sont capables de lier ce stéroïde avec une capacité non significativement différente de celle de la femelle. Cela semble concorder avec ce qui fut décrit pour l'Homme, chez lequel la concentration en CBG n'est pas significativement différente de celle de la Femme, bien que le taux de progestérone soit notablement plus faible (in WestPhal, I97 I ; Furuyama et Nugent, I97I).

Reçu pour publication en janvier 1976. 


\title{
ADDENDUM
}

Cochér et Chambaz (I976) ont récemment identifié par migration électrophorétique de plasma de poussin, en utilisant des conditions expérimentales proches des nôtres (gel de polyacrylamide de $7,5 \mathrm{p}$. Ioo, tampon Tris-glycine $\mathrm{pH} 8,9$ ) une fraction protéique principale capable de lier la corticostérone; cette fraction possède une capacité de liaison limitée et a été assimilée à la CBG par ces derniers auteurs. Elle correspond à la fraction I du profil électrophorétique que nous donnons (fig. 2).

Соснет C., СнамваZ E. M., 1976. High affinity binding of dexamethasone to chicken plasma proteins : some characteristics of chicken corticosteroid binding globulin. Comp. Biochem. Physiol., 58 B, 73-76.

\section{SUMMARY}

\author{
RELATIONSHIP BETWEEN PROGESTERONE BINDING PROTEINS \\ AND PLASMA PROGESTERONE CONTENT IN GALLUS DOMESTICUS
}

$\mathrm{C}_{21}$ steroid-plasma protein interaction is studied in Gallus domesticus. Progesterone and corticosterone bind with high affinity $\left(\mathrm{K}_{\mathrm{A}} \simeq 5 \times 10^{8} \mathrm{M}^{-1}\right)$ and limited capacity $\left(\mathrm{N} \simeq 2 \times 10^{-7} \mathrm{M}\right)$ to the same plasma proteins. Binding capacity is similar in " standard "hens, dwarf hens and roosters $(p>0.05)$. Plasma progesterone concentrations are determined by radioimmunoassay. Values in the females range between 0.3 and $1.2 \mathrm{ng} / \mathrm{ml}$ a few hours after laying (differences between the two types of hens are not significant) and below detection limit in males. No correlation between plasma progesterone content and progesterone protein-binding capacity is observed between hens or between females and males.

\section{RÉFÉRENCES BIBLIOGRAPHIQUES}

Corvol P., Bardin C. W., 1973. Species distribution of Testosterone Binding Globulin. Biol. Reprod., $8,277-282$.

Daniel J. Y., Assernmacher I., 1974. Hormonal effects on plasma binding capacity in ducks. $J$. steroid biochem., 5, 399, Fourth intern. cong. hormo. steroids ; Mexico.

Daughaday W. H., 1956. Evidence for two corticosteroid binding systems in human plasma. J. Lab. clin. Med., 48, 799-808.

DAughaday W. H., I958. Binding of corticosteroids by plasma proteins. IV. The electrophoretic demonstration of corticosteroid-binding globulin. J. clin. Invest., 37, 519-523.

Davis B. J., I962. Disc Electrophoresis, preprinted by Distillation Products Industries, Eastman Kodak Co.

DoE R. P., SeAL U. S., 1963. Protein binding of cortisol in man. J. clin. Invest., 42, 929-940.

FurR B. J. A., 1973. Radioimmunoassay of progesterone in peripheral plasma of the domestic fowl in various physiological states and in follicular venous plasma. Acta endocrinol. (Kbh), $72,89-100$.

Furr B. J. A., Bonney $\quad$ R. C., England R. J., Cunningham F. J., r973. Luteinizing hormone and progesterone in peripheral blood during the ovulatory cycle of the hen. $J$. Endocr., 57, 159-169.

Furuyama S., Nugent C., I971. A radioimmunoassay for plasma progesterone. Steroids, 17, 663-674.

Gould N. R., SIEGEL H. S., 1974. Age variation in corticosteroid binding by serum proteins of growing chickens. Gen. Comp. Endocr., 24, 177-182.

Heyns W., Van Baelen H., De Moor R. P., r967. Study of steroid-protein binding by means of competitive adsorption : application to cortisol binding in plasma. Clin. Chim. Acta, 18, 36I-370.

Kalckar H. M., I947. Differential spectrophotometry of purine componnds by means of specific enzymes. III. Studies of enzymes of purine metabolism. J. Biol. Chem., 167, $46 \mathrm{I}-475$. 
Lagü̈ P. C., Van Tienhoven A., Cunningham F. J., r975. Concentrations of estrogens, progesterone and LH during the ovulatory cycle of the laying chicken (Gallus domesticus). Biol. Reprod., 12, $590-598$.

LoRenz F. W., r969. Reproduction in domestic fowl in Reproduction in domestic animals $\mathrm{H}$. H. CoLE and P. T. Cuprs, Ed., Acad., Press N. Y., Lond., 569-606.

Martra B., 1974. Recherches sur la liaison des stéroides aux protéines dans le sérum de Vertébrés non mammaliens. Thèse Sciences Naturelles, no A o 9967, C.N.R.S.

Martin B., Ozon R., r975. Steroid-Protein interactions in non mammalian Vertebrates. II. Steroids binding proteins in the serum of Amphibians; a physiological approach. Biol. Reprod., 13, 371-380.

Patterson M. S., Green R. C., I965. Measurement of low energy beta emitters in aqueous solution by liquid scintillation counting of emulsions. Analyt. Chem., 37, 854-857.

Raynaud J. P., r973. A computer program for the analysis of binding experiments. Computer Programs in Biomedicine, 3, 63-78.

Rosenthal H. E., I967. A graphic method for the determination and presentation of binding parameters in a complex system. Analyt. biochem., 20, 525-532.

Sandberg A. A., Rosenthal H., Schneider S. L., Slaunwhite W. R., Jr., I966. Protein-steroid interactions and their role in the transport and metabolism of steroids. In : Steroid Dynamics. G. PINcus, T. Nakao and J. F. TAIT. Academic Press, New York, I-6r.

Sandor T., 1972. Corticosteroids in Amphibia, Reptilia and Aves. In : Steroids in non-mammalian Vertebrates, Ed, Inler D. R., Academic Press, New York and London, 253-327.

SCATChard G., I949. The attractions of proteins for small molecules and ions. Ann. N. Y. Acad. Sci. 51, 660-672.

Seal U. S., DoE R. P., 1965. Vertebrate distribution of corticosteroid-binding globulin and some endocrine effects on concentration. Steroids, 5, 827-84I.

Shahabi N. A., Norton H. W., Nalbandev A. V., I975. Steroid levels in follicles and the plasma of hens during the ovulatory cycle. Endocrinology, 96, 962-968.

Sherman M. R., Corvol P. L., O'Malley B. W., r97o. Progesterone-binding components of chick oviduct. I. Preliminary characterization of cytoplasmic components. J. Biol. Chem., 245, 6085-6096.

Shodono M., Nakamura T., Tanabe Y., Wakabayashi K., I975. Simultaneous determinations of oestradiol-1 $7 \beta$, progesterone and luteinizing hormone in the plasma during the ovulatory cycle of the hen. Acta endocrinol. (Kbh), 78, 565-573.

Steeno O., De Moor P., I966. The corticosteroid binding capacity of plasma transcortin in Mammals and Aves. Bull. Soc. Roy. Zool. Anvers, 38, 3-24.

Thibier M., Castanier M., Tea N. T., Scholler R., 1973. Concentrations plasmatiques de la I7 $\alpha$-hydroxy-progestérone au cours du cycle de la Vache. C. $R$. Acad. Sc. Paris, Sér. D., 276, 3049-3052.

Westphal U., I97r. Steroid-protein interactions; in : Monographs on Endocrinology, vol. IV, SpringerVerlag, Berlin. 